\title{
Time to Recovery and Determinants of Trauma Among Patients Admitted to Lemlem Karl Hospital Maichew, Southern Tigray, Ehiopia: A Retrospective Cohort Study
}

\author{
Reda Haddish Berhe ${ }^{1, *}$, Getachew Redae², Yasin Jemal², Abdukadir Nigus Mohammed ${ }^{3}$ \\ ${ }^{1}$ Department of Health Management Information System at Lemlem Karl Hospital, Maichew, Ethiopia \\ ${ }^{2}$ Department of Environmental Sciences, School of Public Health, Mekelle University, Mekelle, Ethiopia \\ ${ }^{3}$ Department of Health Management Information System at Alamata General Hospital, Alamata, Ethiopia \\ Email address: \\ redahaddish@gmail.com (R. H. Berhe), abdukadirnigus@gmail.com (A. N. Mohammed), abdukdir.nigus@mu.edu.et (A. N. Mohammed) \\ ${ }^{*}$ Corresponding author
}

\section{To cite this article:}

Reda Haddish Berhe, Getachew Redae, Yasin Jemal, Abdukadir Nigus Mohammed. Time to Recovery and Determinants of Trauma Among Patients Admitted to Lemlem Karl Hospital Maichew, Southern Tigray, Ehiopia: A Retrospective Cohort Study. International Journal of Biomedical Science and Engineering. Vol. 8, No. 2, 2020, pp. 11-18. doi: 10.11648/j.ijbse.20200802.12

Received: May 5, 2020; Accepted: June 9, 2020; Published: June 20, 2020

\begin{abstract}
Background: Ethiopia is one of the worst countries in the world where trauma especially traffic accident kills a large number of road users every year. In Ethiopia, the magnitude and survival time to recovery of trauma was not well understood. This study intended to examine time to recovery from trauma and predictors among inpatients in Lemlem Karl Hospital, Maichew, Southern Tigray, Ethiopia, 2017. Methods: A retrospective cohort study was conducted by reviewing records of traumatic patients who were admitted to inpatient surgical ward from January 2013 to December 2017 in Lemlem Karl general hospital Maichew town, Tigray, Ethiopia. A total of 327 study participants were selected using simple random sampling. Kaplan-Meier survival plots were used to calculate the crude effect on trauma, survival probabilities and compared using the log-rank test. Cox proportional hazard regression model was used to identify predictors of hospital length of stay. A total of $93.8 \%$ of patients were recovered during the follow-up period with a median time of 4 days. Hence, those who had Complications were about $85 \%$ times less likely to develop complications than those who had no complications (HR $=0.15$; CI 0.09-0.24). In addition to those who get treatment before admission to the ward had 3 times more likely to recover than those patients who didn't get treatment before admission ( $\mathrm{HR}=3.29$; CI: 1.82- 5.95). Therefore, Severity of trauma, number of traumatic organs and treatment given before admission were found significant predictors of time to recovery from trauma. This study provides greater insight of benefits by develop countermeasures that could reduce the number and severity of trauma length of stay.
\end{abstract}

Keywords: Cox Proportional Hazards Model, Length of Stay, Survival, Time to Recovery, Ethiopia

\section{Introduction}

Trauma is a physical damage that results when a human body is subjected to high levels of energy. It can be a bodily lesion resulting from acute exposure to energy in amounts that exceed the threshold of physiological tolerance [1]. It is the major cause of mortality, disability and morbidity among reproductive age group populations in the developing countries and in the world $[2,3]$. Trauma also contributes to about $10 \%$ of disease burden worldwide [4]. Among African nations the rate of injury mortality in 2004 was the highest in
Nigeria followed by South Africa and Ethiopia as the second and third, respectively. However, the rate of trauma mortality was the lowest in Egypt. Africa has the world's highest death rate per population (28.3 per 100,000 of the population)[8] and according to world health organization (WHO) Ethiopia is one of the worst countries in the world where trauma especially traffic accident kills a large number of road users every year [5].

In the year (2014/2015), about 15,086 accidents happened in Ethiopia which caused the losses of 2,161 lives and over ETB 82 million (cost estimate of property damage). From the 
total accidents 15,086 ; serious injury $(2,156)$, fatal $(2,161)$. Of the total 9,$301 ; 913(9.82 \%)$ of them occurred in Tigray. [6]. In Arba Minch general hospital; Road traffic accidents and personal violence were the major cause of injury of all trauma followed by falling accident [7] and in Addis Ababa Ethiopia, hospital-based data indicated that unintentional trauma made up $77 \%$ of pediatric attendees. Burns and traffic injuries were the commonest injury types and cars accidents account $26 \%$ of the road traffic trauma cases [8]. The average hospital length of stay (LOS) in Ethiopia and at national level for inpatients should be 5 days. But Ethiopian monitoring hospital performance showed that, the average of stay for inpatient was 6.7 days-in Ethiopia and 4.7 days in Tigray. But in LKH it was found those 6.6 days. $[18,19]$. LOS has been identified as one of the major drivers of resource consumption in multiple ways. Hospital cost increases because beds and human personnel are occupied by prolonged patients and because of the rise in associated adverse events. In addition, there is a societal cost due to prolonged patients' lost economic productivity [10]. In Ethiopia, the magnitude and survival time to recovery of trauma was not well understood. Therefore, this study aimed to examine time to recovery from trauma and predictors among inpatients in Lemlem Karl Hospital, Maichew, Southern Tigray, Ethiopia, 2017.

\section{Methods}

\subsection{Study Area and Period}

Lemlem Karl Hospital is (LKH) found in Maichew located at $665 \mathrm{~km}$ north of Addis Ababaand $130 \mathrm{~km}$ south of Mekelle. The hospital gives service for over one million people in the Southern Tigray and vicinity areas of Afar and Amhara regions for basic and referral services with a total capacity of about 132 beds. Currently the hospital is staffed with a total of $339 ; 199(58.7 \%)$ technical and140 (41.3\%) supportive. It provides a range of services such as; outpatient, inpatient, maternity and emergency services. This study was conducted in surgical ward of Lemlem Karl hospital Maichew from January 05, 2018- February 05, 2018.

\subsection{Study Design and Population}

A retrospective cohort study was conducted for traumatic patients admitted at surgical ward of LKH from January 2013-December 2017. The Source population was all patients admitted due to trauma in LKH Maichew. Study population was all patients who were admitted in the inpatient surgical department of LKH due to trauma from January 2013December 2017. The traumatic patients who were admitted in surgical ward of LKH from January 2013- December 2017 were randomly selected. All traumatic patients admitted in surgical ward from January 2013- December 2017 were included in the study. However, incomplete medical record and those who had less than 24 hour's length of stay were excluded from the study.

\subsection{Sample Size and Sampling Procedure}

A "Simple random sampling" technique was applied to collect the most representative samples according to guidelines of sampling techniques stated on APHA [39]. A total of 327 samples were collected from sampling site in the study period.

\subsection{Data Collection Methods and Tools}

The main outcome variables were survival time to recovery from trauma, the patient length of stay in hospital 'leading to recovery' (days) was considered as a survival variable and was calculated by subtracting the date of admission from date of discharge. The patients discharged from the hospital without recovery, those who had been left against medical advice (refused treatment), and died was determined as censored data. Recovery of patients was the event of interest and the coding was " 1 " for recovered and " 0 " for censored. Predictor variables were sociodemographic factors, characteristics of trauma, clinical and management factors.

Data was collected using a pre tested structured checklist by adopting from the world health organization's trauma surveillance guideline and literatures [1, 12, 15, 35] after customized according to the variables available in the registration book. Demographic characteristics of the patients, clinical factors, management related factors and characteristics of trauma were the variables included. We extracted data on these predictor variables from register, follow-up forms, and other clinical records. These registers and forms are regularly filled as part of a routine paper-based patient record system. The data were extracted by a trained nurse.

\subsection{Data Processing and Analysis}

Data was entered, cleaned, recoded using SPSS Ver-20 software and was analyzed using STATA ver-12. Descriptive statistics was used to summarize the characteristics of the cohort. Kaplan-Meier survival plots were used to calculate the crude effect on trauma patients free survival probabilities and compared using the log rank test. Crude analysis was used for inclusion of variables into multivariable analysis with cut off $p$-value $\leq 0.25$. To identify potential predictors of hospital length of stay, Cox proportional hazard regression model was employed and p-value $<=0.05$ declares the significance of the variables at $95 \%$ confidence level. Model building techniques (Test the interaction of covariates with time and Cox-Snell residual) are necessary to choose the best fitted model. The test of interaction covariates should be time-dependent if the test shows the interactions significantly exist, which means the proportional assumption is violated. The standard cox analysis follows, by use of the produced product of the time variable ( $\mathrm{T}_{-} \mathrm{Cov}$ ) ) and that same variable alone. 'If ( $\mathrm{T} \_$Cov_) is above 0.05 , the proportional hazard assumption is satisfied. For Cox-Snell residual, the line more closes to bisector of the first quarter, used as the indicator of the best model. 


\section{Results}

\subsection{Baseline Characteristics of Trauma Among Admitted Patients}

A total of 320 records of trauma patients were reviewed and analyzed with response rate of $98 \%$. Two hundred thirty three $(72.80 \%)$ of the records belong to males and 223 $(69.70 \%)$ of the total participants were from rural areas. From the 320 included total participants $300(93.8 \%)$ of the patients reported full recovery at a median time of 4 days. (Table 1)

Table 1. Background characteristics of trauma patients and summary of the study clinical variables treated at Lemlem Karl Hospital maichew from January 2013-December $2017(n=320)$.

\begin{tabular}{|c|c|c|c|c|c|}
\hline Variable names & Category & $\begin{array}{l}\text { Median (IQR) time to } \\
\text { recovery (days) }\end{array}$ & Count (\%) & Events & Censored \\
\hline \multirow{4}{*}{ Age } & $<5$ years & $2(3)$ & $9(2.8 \%)$ & 8 & 1 \\
\hline & $5-14$ years & $7(11)$ & $68(21.3 \%)$ & 62 & 5 \\
\hline & $15-44$ & $4(6)$ & $182(56.9 \%)$ & 173 & 9 \\
\hline & $45-64$ years $\&>64$ & $16(25)$ & $61(19.1 \%)$ & 56 & 5 \\
\hline \multirow{2}{*}{ Sex } & Male & $4(8)$ & $233(72.8 \%)$ & 218 & 15 \\
\hline & Female & $4(8)$ & $87(27.2 \%)$ & 82 & 5 \\
\hline \multirow{2}{*}{ Residence } & Rural & $4(9)$ & $223(69.7 \%)$ & 205 & 18 \\
\hline & Urban & $3(6)$ & $97(30.3 \%)$ & 95 & 2 \\
\hline \multirow{2}{*}{ Co morbidity } & Yes & $22(12)$ & $9(2.80)$ & 7 & 2 \\
\hline & No & $4(8)$ & $311(97.20)$ & 293 & 18 \\
\hline \multirow{3}{*}{ Number of co morbidities } & No co morbidity & $4(7)$ & $311(97.20)$ & 293 & 17 \\
\hline & One co morbidity & $22(7)$ & $5(1.60)$ & 3 & 2 \\
\hline & $>=2$ co morbidities & $22(7)$ & $4(1.20)$ & 4 & 1 \\
\hline \multirow{3}{*}{ Complications } & Yes & $18(19)$ & $59(18.10)$ & 46 & 13 \\
\hline & No & $3(4)$ & $261(81.90)$ & 254 & 7 \\
\hline & Not applicable (NA) & $4(7)$ & $290(90.60)$ & 271 & 11 \\
\hline \multirow{3}{*}{$\begin{array}{l}\text { Time taken to surgery after } \\
\text { admission }\end{array}$} & $0-24$ hrs. & $5(5)$ & $21(6.70)$ & 20 & 5 \\
\hline & $25-48 \mathrm{hrs}$. & $18(6)$ & $4(1.30)$ & 4 & 2 \\
\hline & $>48$ hrs. & $41(40)$ & $5(1.60)$ & 5 & 2 \\
\hline \multirow{3}{*}{ Type of trauma } & Unintentional & $4(8)$ & $228(71.30)$ & 213 & 15 \\
\hline & Intentional & $5(7)$ & $92(28.80)$ & 87 & 5 \\
\hline & Interpersonal & $5(7)$ & $90(28.10)$ & 85 & 5 \\
\hline \multirow[t]{2}{*}{ Type of intentional trauma } & Self-harm & $5(7)$ & $2(0.60)$ & 2 & 0 \\
\hline & NA & $5(7)$ & $228(71.30)$ & 213 & 15 \\
\hline \multirow{8}{*}{ Nature of trauma } & Fracture & $4(8)$ & $216(67.50)$ & 200 & 12 \\
\hline & Sprain, strain & $3(10)$ & $11(3.40)$ & 11 & 2 \\
\hline & Bruise or superficial & $2(1)$ & $9(2.80)$ & 9 & 2 \\
\hline & Burns & $5(6)$ & $24(7.50)$ & 23 & 1 \\
\hline & Cuts, bites or open wound & $8(8)$ & $37(11.60)$ & 34 & 3 \\
\hline & Organ system trauma & $3(6)$ & $12(3.80)$ & 12 & 0 \\
\hline & Concussion & $2(1)$ & $1(0.30)$ & 1 & 0 \\
\hline & Others & $2(1)$ & $10(3.10)$ & 10 & 0 \\
\hline \multirow{9}{*}{ Mechanism of Trauma } & Traffic accident & $4(6)$ & $107(33.40)$ & 101 & 6 \\
\hline & $\begin{array}{l}\text { Blunt trauma, Stab, Stuck/hit by } \\
\text { person }\end{array}$ & $4(6)$ & $94(29.40)$ & 89 & 5 \\
\hline & Fall, Stuck/hit by object & $4(13)$ & $71(22.20)$ & 64 & 7 \\
\hline & Fire, flames or heat & $5(6)$ & $24(7.50)$ & 23 & 1 \\
\hline & Poisoning & $5(6)$ & $4(1.30)$ & 4 & 0 \\
\hline & Gun shot & $3(3)$ & $1(0.30)$ & 1 & 0 \\
\hline & Others & $2(0)$ & $19(5.90)$ & 18 & 1 \\
\hline & Moderate & $3(5)$ & $273(85.00)$ & 269 & 4 \\
\hline & Severe & $20(13)$ & $47(15)$ & 31 & 16 \\
\hline
\end{tabular}

IQR: Inter Quartile Range, LOS: Length of stay.

\subsection{Clinical and Management Related Characteristics}

Concerning to the type of trauma, from the total participants $228(71.30 \%)$ of them had unintentional trauma. Of all the participants $65(20.30 \%)$ of them had multiple organ failures and 47 (15\%) of were with severity of trauma. Our finding also found that 9 (2.80\%), 59 $(18.10 \%)$ had co morbidities and complications respectively. Regarding the nature of trauma non recovery was common among patients experienced fracture (16 from 216) and from mechanisms of trauma traffic accident account 107 (33.40\%). Of all the participants 305 (95.30\%) of them had got treatment before admission either at the emergency outpatient department or at their referring site (table 1). From the total nature (diagnosis) of trauma fracture holds $216(67.50 \%)$ and regarding the site of trauma $126(39.40 \%)$ belongs to head trauma. 


\subsection{Model Building Techniques (Test the Interaction of Covariates with Time, Cox-Snell Residual)}

Regards to the present study, the covariates timedependent test shows the interactions significantly exist, which means the proportional assumption is violated. The standard cox analysis follows, by use of the produced product of the time (T_Cov_) was above 0.05 (which is 0.08 ), hence, the proportional hazard assumption is satisfied. In addition to, For Cox-Snell residual shows that, the line was more closes to bisector of the first quarter, used as the indicator of the best model.

Table 2. Background characteristics of trauma patients and summary of the study clinical variables treated at Lemlem Karl Hospital maichew from January 2013-December $2017(n=320)$.

\begin{tabular}{|c|c|c|c|c|c|}
\hline Variable & Category & Median (IQR) LOS (days) & $\mathbf{N}(\%)$ & Events & Censored \\
\hline \multirow{3}{*}{ GCS } & mild (13-15) & $3(4)$ & $135(42.20)$ & 132 & 3 \\
\hline & moderate $(9-12)$ & $4(8)$ & $147(46)$ & 139 & 8 \\
\hline & Severe $(<8)$ & $19(12)$ & $38(11.80)$ & 29 & 9 \\
\hline \multirow{3}{*}{ Level of consciousness } & Normal & $3(5)$ & $238(74.40)$ & 231 & 7 \\
\hline & Obtunded & $11(16)$ & $76(23.75)$ & 65 & 11 \\
\hline & Comatose & $22(1)$ & $6(1.95)$ & 4 & 2 \\
\hline Managements given & Conservative & $4(8)$ & $295(92.20)$ & 275 & 20 \\
\hline \multirow{2}{*}{ Rx given before admission } & Yes & $4(7)$ & $305(95.30)$ & 286 & 19 \\
\hline & No & $12(12)$ & $15(4.70)$ & 14 & 1 \\
\hline \multirow{2}{*}{ Previous history of trauma } & Yes & $9(17)$ & $13(4.10)$ & 11 & 2 \\
\hline & No & $4(8)$ & $307(95.90)$ & 289 & 18 \\
\hline \multirow{3}{*}{ Site of trauma } & Head & $4(7)$ & $126(39.40)$ & 113 & 13 \\
\hline & Extremities & $5(9)$ & $74(23.10)$ & 73 & 1 \\
\hline & Others & $4(7)$ & $120(35.50)$ & 114 & 6 \\
\hline Number of traumatic organs & Multiple & $17(17)$ & $65(20.30)$ & 54 & 11 \\
\hline \multirow{2}{*}{ Vital sign at arrival } & Yes & $4(8)$ & $318(99.4)$ & 299 & 19 \\
\hline & No & $9(0)$ & $2(0.6)$ & 1 & 1 \\
\hline \multirow{5}{*}{ Types of investigations done } & Imaging & $2(3)$ & $1(0.30)$ & 1 & 0 \\
\hline & Clinical & $2(3)$ & $15(4.70)$ & 14 & 1 \\
\hline & Laboratory\& Clinical & $4(6)$ & $57(17.80)$ & 56 & 1 \\
\hline & Imaging \& Clinical & $3(2)$ & $42(13.10)$ & 40 & 2 \\
\hline & Laboratory, Clinical \&Imaging & $5(9)$ & $205(64.60)$ & 189 & 16 \\
\hline \multirow{2}{*}{ Discharge condition (Status) } & $1=$ Improved (Event) & & $93.80 \%$ & 300 & - \\
\hline & $0=$ Censored (Dead, Referred, R & ed treatment) & $6.30 \%$ & - & 20 \\
\hline \multirow{3}{*}{$\begin{array}{l}\text { LOS (time to recovery) in } \\
\text { days }\end{array}$} & $<=5$ days & & $74(23.10)$ & 65 & 9 \\
\hline & 6-10 days & & $120(37.50)$ & 114 & 6 \\
\hline & $>10$ days & & $126(39.40)$ & 121 & 5 \\
\hline
\end{tabular}

GCS: Glasgow coma score; IQR: Inter quartile range, LOS: Length of Stay, Rx: Treatment.
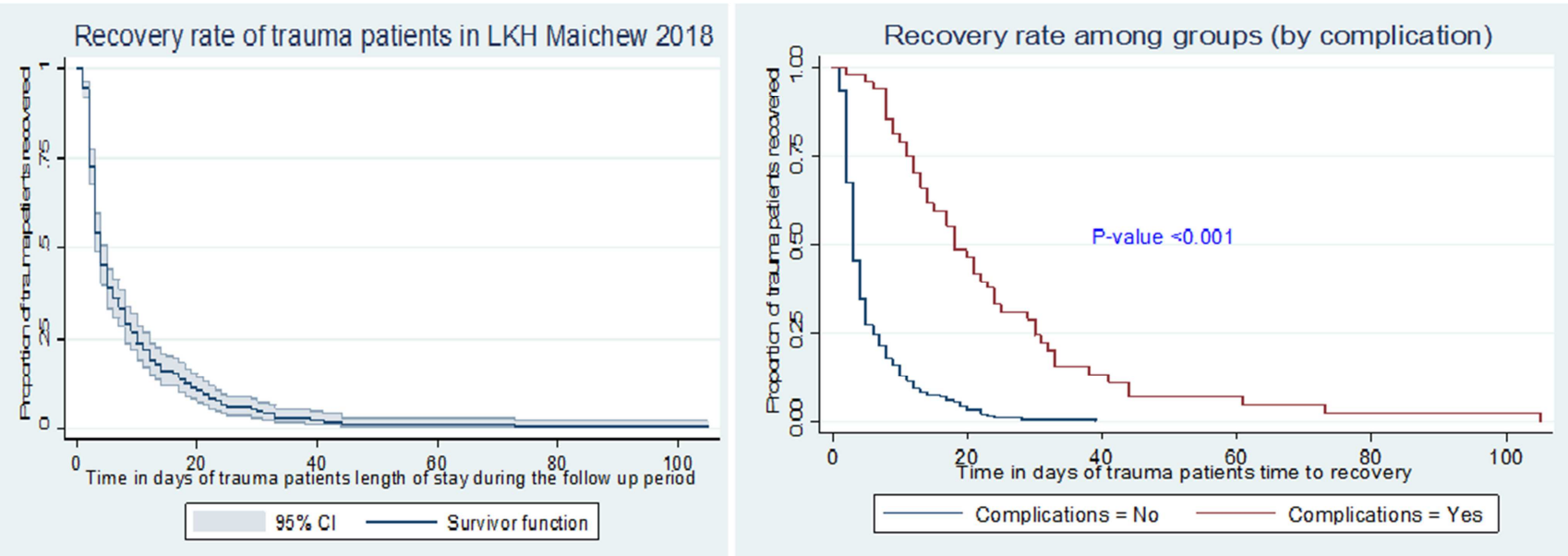

Figure 1. Kaplan-Meier recovery and survival estimate of trauma patients by the presence of complications treated at Lemlem Karl Hospital from January 2013-December 2017.

The graph shows the proportion of trauma patients who survived during the follow up recovery period. Accordingly, as can be seen from the graph; during the first 20 days the graph went down gradually which shows a higher proportion of patients were had longer stay and there was a lower probability of faster recovery. While, over the next 20 days (20 to 40), the proportion of LOS has slightly increased and the graph fell down slowly up to the third follow up time 
(60day). In the last follow up period the graph became straight which indicates the proportion of traumatic patients recovered remained stable indicating virtually no longer stay. (Figure 1)

In the next graph (Figure 1), we see that the survival function for each group of complications is not perfectly parallel but separate except at the very beginning. The overlap at the very beginning should not cause too much concern because it is determined by only a very few number of censored subjects. In general, the log-rank test places the more emphasis on differences in the curves at larger time values. Curves do not cross each other and the hazards are proportional. Therefore, they are good candidates to enter in the cox-regression model. Patients with complication have lower recovery probabilities (takes more time to recover) than patients without complication.

\subsection{Magnitude of Time to Recovery of Trauma Among Admitted Patient}

Patients were followed for a median of 4 days with a total of 2493 person time of follow up and the total incidence (new recovery rate per 100 person times) was $12 \%$ (95\% CI: $(0.11-0.14))$. The slower age groups recovery was among $>64$ age groups and having a median time of 12 days with Inter Quartile Range of 18 (table 3). The median (IQR) recovery time for patients who had co morbidity were 22 (12), Complications were 18 (19), patients did not get treatment before admission were 12 (12) and patients with multiple organ failure were 17 (17) (table 3).

Table 3. Time to recovery and determinants in a cohort of trauma among patient admit to Lemlem Karl Hospital maichew from 2013-2017 (n=320).

\begin{tabular}{|c|c|c|}
\hline Variables & CHR [95\% CI] & AHR $[95 \% \mathrm{CI}]$ \\
\hline \multicolumn{3}{|l|}{ Complications } \\
\hline Yes & $4.7[3.3,6.8] * * * *$ & $0.15[0.09,0.24] * * * *$ \\
\hline No & Reference & Reference \\
\hline \multicolumn{3}{|l|}{ Severity of trauma } \\
\hline Severe & $0.35[0.25,0.5] * * * *$ & $0.15[0.08,0.27] * * * *$ \\
\hline Moderate & Reference & Reference \\
\hline \multicolumn{3}{|l|}{$\begin{array}{l}\text { Treatment given } \\
\text { before admission }\end{array}$} \\
\hline Yes & $0.37[0.2,0.66] * * * *$ & $3.29[1.82,5.95] * * * *$ \\
\hline No & Reference & Reference \\
\hline \multicolumn{3}{|l|}{$\begin{array}{l}\text { Number of } \\
\text { traumatic organs }\end{array}$} \\
\hline Multiple & $0.35[0.26,0.49] * * * *$ & $0.42[0.30,0.60] * * * *$ \\
\hline Single & Reference & Reference \\
\hline \multicolumn{3}{|l|}{ Complications\# } \\
\hline \multicolumn{3}{|l|}{ Severity of trauma } \\
\hline Yes \# Severe & $3.68[0.26,0.49]^{* * *}$ & $7.15[2.97,17.19] * * * *$ \\
\hline
\end{tabular}

Significant at: $* \mathrm{P}<0.25 ; * * \mathrm{P}<0.05 ; * * * \mathrm{P}<0.01$; **** $\mathrm{P}<0.001$; CHR: Crude hazard Ratio; AHR: Adjusted hazard Ratio; CI: Confidence Interval.

\subsection{Determinants of Time to Recovery of Trauma Among Admitted Patient}

This study revealed that the patients with lower frequency recovery of trauma had higher rate of survival. Complications, treatment given before admission and number of traumatic organs were significantly associated with frequency recovery of trauma. Hence, those who had Complications were about $85 \%$ times less likely to develop complications than those who had no complications $(\mathrm{HR}=0.15$; CI 0.09-0.24). In addition to those who get treatment before admission to the ward had 3.29 times more likely to recover than those patients who didn't get treatment before admission ( $\mathrm{HR}=3.29$; $\mathrm{CI}$ : 1.82- 5.95). Number of traumatic organs $(\mathrm{HR}=0.42$; $\mathrm{CI} 0.30-0.60)$ implies, as the number of traumatic organs of a patient increases and all other variables are held constant, the rate of recovery decreases by $58 \%$ or patients with multiple traumatic organs recovered at a rate $58 \%$ slower than those patients with single traumatic organs.

Severity of trauma $(\mathrm{HR}=0.15$; CI: $0.08-0.27)$ implies, patients with severe trauma recovered at a rate of $85 \%$ slower than those patients with moderate traumas. Additionally the risk of long hospital stay was found to higher the hazard of developing complications by 7 times (HR: 7.15; 95\% CI: $2.97-17.19$ ) for patients with severe traumas as compared to patients having complications with moderate trauma.

\section{Discussion}

This study employed survival analysis to examine posttrauma recovery time and its predictors. The findings revealed that $93.8 \%$ of patients achieved recovery during the follow-up period and the overall hospital LOS ranged 1 day to 105 days. In addition the total median time to recovery was 4 days. Of the participants experienced recovery $74(23.40 \%)<=5$ days of them were recovered less than or equal to five days after trauma, $120(35.30 \%)$ of them 6-10 days and $126(41.30 \%)$ of them had $>10$ days. Additionally, in our study severity of trauma, presence of complications, treatment given before admission and number of organ failures were found as significant predictors of time to recovery from trauma. This finding is congruent with the cohort studies done in Canada experiencing from all traumas reported a recovery rate of 92.72\% during two months [22] and study done in British Colombia experiencing hip injury reported $92.1 \%$ recovery rate [35]. A study done in Norway experiencing from all traumas reported a recovery rate of $87 \%$ [11]. Even if these countries are highly developed which are not comparable to our setups related to quality of care, the reason of higher recovery rate found in our study may be due to most of the patients $(95.3 \%)$ included in our study had got treatment before they were admitted to the ward.

A study in New Delhi (India) experiencing from brain traumas showed that the average duration of hospital stay was 5 days [23]. Similar studies conducted in Kenya experiencing from all traumas also found that $93.1 \%$ at two weeks [20] and 92.85\% [29] of their participants recovered after trauma. In addition, at a tertiary teaching hospital in Lusaka, Zambia experiencing from all traumas the median length of stay for all admitted patients due to trauma was 6.6 days [38] the recovery rate of a cross-sectional study conducted in Ethiopia Wolaita Zone was $81.5 \%$ at a median time of 5 days [39] which are almost similar to our study. 
However, study done in Rwanda, Kigali reported recovery rate of $81.4 \%$ at a median time of 16 days [36] different from our study. A study conducted in South Africa from experiencing burn injuries [28], Egypt from experiencing Kidney injuries [27] and Cameron from experiencing injuries at extremities [32] found that recovery rate of $73.4 \%, 77 \%$, and $74.2 \%$ respectively slightly lower than our study. Moreover, recovery rate in studies conducted in Iran from experiencing head and extremity injuries was 98\% [24] slightly higher than our study.

These conflicting findings can be attributed to the differences in characteristics of the studies such as follow-up period, study population, inclusion criteria, study settings, type \& number of injured organs and as well as patients' access to healthcare services.

This study indicated that; the most commonly affected age group was adults (15 to 64) and this almost congruent with the findings of the previous study done in Mekele Tigray Ethiopia. In addition to this our study reveals that; males were more affected than females $(72.8 \%$ vs. $27.2 \%)$ and is almost similar with previous study in Mekele $(74.3 \%$ vs. $25.7 \%$ ) [15]. This is likely due to the nature of work exposing, the increased level of participation in high-risk activities among male individuals.

From the total participants included to our study (69.7\%) were from rural areas. The most common mechanism of trauma were traffic accident $(33.4 \%)$, blunt trauma, stab, stuck/hit by person (29.4\%) fall, stuck / hit by animal or object $(22.2 \%)$. Among parts of the body mostly affected were head injuries predominated $(39.4 \%)$ which is consistent with other similar studies [12, 14, 15, 17, 20, 24, 29, 36].

Our finding showed that; age was not a significant predictor of time to recovery from trauma. Haghparast-Bidgoli $\mathrm{H}$ et al also reported that age is not a significant predictor for recovery from trauma [12]. However; other studies have reported age as a significant predictor of time to recovery from trauma $[9,11$, 15, 16, 22, 27, 35]. A study conducted by Shahram Bolandparvaz $\mathrm{M}$ et al also found that, age as potential predictor of recovery from trauma [24]. These differences are may be due to the inclusion of age groups to our study; in our study we include all age groups, but most of the other studies include either adult or pediatric age groups. Additionally, in our study most of the participants were in the age of 15-64 (adults) who are expected to have high recovery rate than the other age groups.

This study finding also indicated that; no significant difference between male and female participants regarding recovery rate. Moreover, Cox regression analysis also revealed that gender was not a predictor for time to recovery. This is congruent with the findings of previous studies [21, 24, 27, 28]. However, several other studies reported gender as a predictor for recovery $[12,26,25,26,34]$. This conflicting finding can be attributed to greater proportion of male participants in our study. Recovery rate was also higher among patients residing in rural areas although the difference between these patients and patients living in urban areas was not statistically significant. This is congruent with the findings of previous study [21]. But some studies reported that residence as a predictor for time to recovery $[9,15,22$, 23]. This conflicting finding may be also attributed to greater proportion of participants from rular in our study.

Our finding showed that recovery rate among patients with severe traumas was significantly lower than other patients. Patients with severed traumas usually have a longer hospital stay and recovery. Hence, delayed recovery among them is expected. In the Cox regression analysis severity of trauma was found as a significant predictor for recovery. Some studies also reported trauma severity as a predictor of recovery $[9,11,12,20-22,27,30-33]$. Individuals with isolated traumas had significantly faster recovery rate. Isolated traumas are usually less severe than multiple traumas. Accordingly, these patients achieve recovery in relatively shorter period of time. Ulvik A eta al [11] also found that the probability of early recovery was higher among patients with isolated injuries.

This study finding indicated that; co morbidities and number of co morbidities are not significant predictors of time to recovery. However, previous studies reported it as predictors for recovery from trauma $[9,13,14,22,28,33-$ 35]. This conflicting finding may be attributed to smaller proportion of participants having co morbidities in our study. Presence of complications among individuals was found that as significant predictor of time to recovery and indicated that patients who were developed complications had longer time of hospital stay than those patients who didn't develop complications. This is congruent with the findings of previous studies $[13,20,22,29,33]$ and it is expected.

The Kaplan Meier analysis showed that recovery rate was higher among patients who get treatment before admission. Cox regression analysis also indicated that treatment given before admission was a significant predictor for recovery. In line with our findings, Seidenberg P et al [38] also reported that treatment given before admission was a strong predictor for recovery. Cox regression analysis also revealed that delay to surgery was not a predictor for time to recovery. However, other studies reported as a predictor for recovery [25, 27, 35, 37]. This conflicting finding can be attributed to smaller proportion of participants had got operational management in our study.

Our study also indicated that GCS was not a predictor for time to recovery. This is in line with the cohort study done in Iran [25]. However, other studies reported as a predictor for recovery $[16,22,27,30]$. This conflicting finding may be attributed to proper way of recording GCS of participants scale in our study.

Generally it is difficult to compare length of stay (median time to recovery) between countries, due to differences by the care given in organization of trauma care and variations in patterns of injury. Some of the conflicting findings can be attributed to the differences in characteristics of the studies such as follow-up period, study population, inclusion criteria, and definition of LOS as well as patients' access to healthcare services, health care quality, number of staffs, continuous patient follow up system of the of the hospitals. Therefore, findings in this study should be interpreted in the light of the 
inherent limitations of the study.

\section{Conclusion}

From the total participants included to our study, 300 $(93.80 \%)$ of them were recovered at a median time of 4 days which is almost similar with Ethiopian standard. The findings of the present study showed that; presence of complications, severity of trauma, number of traumatic organs and treatment given before admission were found significant predictors of time to recovery from trauma. This study provides greater insight of benefits by develop countermeasures that could reduce the number and severity of trauma length of stay.

\section{Recommendation}

To regional health bureau and stakeholder:

1. Even though the total hospital length of stay is almost similar to the national standard, there is prolonged length of stay in respective of the specific cases.

2. Severity of trauma, treatment given before admission, presence of complications and number of traumatic organs had an impact on time to recovery, hence, the hospital should work in such a way that in reducing the burdens by following up-to-date guidelines and maintaining standard of care.

To researcher:

1. To assess time to recovery and determinant factors of trauma among admitted patients using stronger study design.

\section{Author Contributions}

RH conceived the study, involved in the data analysis and Report writing and drafted the manuscript. AN was involved in the conception, design, data analysis and review. GR and $\mathrm{YJ}$ reviewed the manuscript. All authors read and approved the manuscript.

\section{Competing Interest}

The authors declare that they have no competing interests.

\section{Acknowledgements}

We thank all staffs of Lemlem Karl Hospital Maichew especially surgical ward staff for their compassionate cooperation and provision accurate information during data collection. We would also like to acknowledge Mekelle University, College of Health Sciences, department of biostatistics for facilitating the study.

\section{References}

[1] Holder Y, Peden M, Krug E, et al. Injury surveillance guidelines: World Health Organization Geneva; 2011.
[2] Mondello S, Cantrell A, Italiano D, et al. Complications of trauma patients admitted to the ICU in level I academic trauma centers in the United States. BioMed research international. 2014; Volume 2014.

[3] Wesson HK, Bachani AM, Wekesa JM, et al. Assessing trauma care at the district and provincial hospital levels: a case study of hospitals in Kenya. Injury. 2013; 44: S75-S80.

[4] Abedzadeh-Kalahroudi M, Razi E, Sehat M, Asadi-Lari M. Return to work after trauma: A survival analysis. Chinese journal of traumatology. 2017; 20 (2): 67-74.

[5] Peden M. World report on child injury prevention: World Health Organization; 2008.

[6] Kebede N. Africa Case Study: Road Safety in Ethiopia United Nations Economic Commission for Africa. 2017 (ECA/NRID/019).

[7] Misker. Magnitude and Factors Associated with Road Traffic Accident among; traumatized Patients in Arba Minch General hospital. Int J Pub Health Safe. 2017; 2 (3).

[8] Tiruneh BT. Factors associated with unintentional injury among the pediatric age population in the hospitals of Amhara National Regional State, Ethiopia.. African Journal of Emergency Medicine. 2017; 7: 55-9.

[9] Tardif P-A, Moore L, Boutin A, Dufresne P, Omar M, Bourgeois G, et al. Hospital length of stay following admission for traumatic brain injury in a Canadian integrated trauma system: A retrospective multicenter cohort study. Injury. 2017; 48 (1): 94-100.

[10] Hwabejire JO, etal. Excessively Long Hospital Stays After Trauma in Massachusetts General Hospital. Are Not Related to the Severity of Illness. JAMA Surg. 2013; 148 (10): 956-61

[11] Ulvik A, Kvåle R, Wentzel-Larsen T, Flaatten H. Multiple organ failure after trauma affects even long-term survival and functional status. Critical Care. 2007; 11 (5): R95.

[12] Haghparast-Bidgoli H, Saadat S, Bogg L, et al. Factors affecting hospital length of stay and hospital charges associated with road traffic-related injuries in Iran. BMC health services research. 2013; 13 (1): 281.

[13] Shekhar C, Gupta LN, Premsagar IC, Sinha M, Kishore J. An epidemiological study of traumatic brain injury cases in a trauma centre of New Delhi (India). Journal of emergencies, trauma, and shock. 2015; 8 (3): 131.

[14] Hailemichael F, Suleiman M, Pauolos W. Magnitude and outcomes of road traffic accidents at Hospitals in Wolaita Zone, SNNPR, Ethiopia. BMC research notes. 2015; 8: 135.

[15] Amdeslasie F, Kidanu M, Lerebo W, Ali D. Patterns of trauma in patient seen at the emergency clinics of public hospitals in Mekelle, northern Ethiopia. Ethiopian medical journal. 2016; 54 (2).

[16] Seid M, Azazh A, Enquselassie F, Yisma E. Injury characteristics and outcome of road traffic accident among victims at Adult Emergency Department of Tikur Anbessa specialized hospital, Addis Ababa, Ethiopia: a prospective hospital based study. BMC emergency medicine. 2015; 15 (1): 10.

[17] Osman M, Kebede Y, Anberbir S. Magnitude and pattern of injuries in north Gondar administrative zone, northwest Ethiopia. Ethiopian medical journal. 2003; 41 (3): 213-20. 
[18] Ghebreyesus TA. Ethiopian Hospital Reform Implementation Guidelines. Hospital Management Initiative. 2010; 1 (1).

[19] Accorsi S, etal. Monitoring hospital performance: the challenge of efficiency in service delivery in ethiopian hospitals. International Pty Ltd. 2010; 3 (1).

[20] Saidi H, Mutiso B. Injury outcomes in elderly patients admitted at an urban African hospital. Surgical Science. 2013; 4 (06): 292.

[21] De la Plata CDM, Hart T, Hammond FM, et al. Impact of age on long-term recovery from traumatic brain injury. Archives of physical medicine and rehabilitation. 2008; 89 (5): 896903.

[22] Bergeron E, Lavoie A, Moore L, et al. Comorbidity and age are both independent predictors of length of hospitalization in trauma patients. Canadian journal of surgery. 2005; 48 (5): 361 .

[23] Bala M, Kashuk JL, Willner D, et al. Looking beyond discharge: clinical variables at trauma admission predict long term survival in the older severely injured patient. World journal of emergency surgery. 2014; 9 (1): 10.

[24] Shahram Bolandparvaz M, Mahnaz Yadollahi M, Hamid Reza Abbasi, MD, et al. Injury patterns among various age and gender groups of trauma patients in southern Iran; A crosssectional study. Medicine. 2017; 96 (41).

[25] Oveis Salehi, Seyed Ashkan Tabibzadeh Dezfuli, Seyed Shojaeddin Namazi, Maryam Dehghan Khalili, Saeedi M. A New Injury Severity Score for Predicting the Length of Hospital Stay in Multiple Trauma Patients; Tehran University of Medical Sciences, Tehran, IR Iran. Trauma Mon. 2016; 21 (1): e20349.

[26] Liu T, Xie J, Yang F, Chen JJ, Li ZF, Yi CL, et al. The influence of sex on outcomes in trauma patients: a metaanalysis. Am J Surg. 2015; 210 (5): 911-21.

[27] Samar AE, Giovanni T, Robert Q, et al. Risk, Predictors, and Outcomes of Acute Kidney Injury in Patients Admitted to Intensive Care Units in Egypt. Scientific reports 2017; 7 (17163).

[28] Lisa Blom, Anders Klingberg, Lucie L, et al. Gender differences in burns: A study from emergency centres in the Western Cape, South Africa; b u rns Elsevier Ltd. 2016; 4 (2): $160-1608$
[29] Saidi H, Macharia W, Ating'a J. Outcome for hospitalized road trauma patients at a tertiary hospital in Kenya. European Journal of Trauma 2005; 31 (4): 401-6.

[30] Fitzharris, Bowman, Ludlow. Factors associated with returnto-work and health outcomes among survivors of road crashes in Victoria. Australian and new zealand journal of public health. 2010; 34 (2).

[31] Samir M, etal. Trauma Center Finances and Length of Stay: Identifying a Profitability Inflection Point: Department of Surgery,. Medical University of South Carolina, Charleston, SC. $2010 ; 210$ (5): 817-21.

[32] Murgatroyd, etal. Predictors of return to work following motor vehicle related orthopedic trauma. BMC Musculoskeletal Disorders. 2016; 17: 171.

[33] M. N. Mnguni DJJM, T. E. Madiba. Abdominal Trauma in Durban, South Africa: Factors Influencing Outcome. Int Surg 2012; 97: 161-8.

[34] B W, A SP. prediction model for length of stay in Florida hospitals: Big data analysis. Journal of Basic and Applied Research Internationa 2015; 8 (4): 304-13.

[35] Lefaivre K, Macadam S, Davidson D, et al. Length of stay, mortality, morbidity and delay to surgery in hip fractures. Bone \& Joint Journal. 2009; 91 (7): 922-7.

[36] Mbanjumucyo G, George N, Kearney A, et al. Epidemiology of injuries and outcomes among trauma patients receiving prehospital care at a tertiary teaching hospital in Kigali, Rwanda. African journal of emergency medicine. 2016; 6 (4): 191-7.

[37] Jayaraman S, Mabweijano J, Mijumbi C, etal. The Care of Injured Patients Admitted to Mulago National Referral Hospital in Kampala, Uganda Emerg Med (Los Angel). 2015; 5: 270 .

[38] Seidenberg P, Brown RO, Cerwensky K, et al. Epidemiology of Injuries, Outcomes, and Hospital Resource Utilization at a Tertiary Teaching Hospital in Lusaka, Zambia. Academic Emergency Medicine. 2013; 20: S325.

[39] Fox J, Weisberg S. Cox Proportional-Hazards Regression for Survival Data. Second Edition ed. USA: Cohort to Applied Regression; 2011. 